\title{
Tolerability of and Adherence to Topical Treatments in Atopic Dermatitis: A Narrative Review
}

\author{
Heather L. Tier (D) - Esther A. Balogh • Arjun M. Bashyam • \\ Alan B. Fleischer Jr · Jonathan M. Spergel • E. J. Masicampo • \\ Lara K. Kammrath · Lindsay C. Strowd · Steven R. Feldman
}

Received: January 7, 2021 / Published online: February 18, 2021

(C) The Author(s) 2021

\section{ABSTRACT}

Atopic dermatitis (AD) is a common, chronic inflammatory skin disease that oftentimes requires complex therapy. Poor adherence is a major barrier to $\mathrm{AD}$ treatment success. An interspecialty, virtual roundtable panel was held, through which clinical dermatologists, allergists, and behavioral and social psychologists discussed $\mathrm{AD}$ management and adherence. Relevant literature was reviewed, and the content of this article was organized based on the roundtable discussion. Current guidelines for $\mathrm{AD}$ treatment include maintenance and acute

H. L. Tier $(\bowtie)$ E. A. Balogh · A. M. Bashyam . A. B. Fleischer Jr · L. C. Strowd · S. R. Feldman Department of Dermatology, Center for

Dermatology Research, Wake Forest School of

Medicine, Medical Center Boulevard, Winston-

Salem, NC 27157-1071, USA

e-mail: tier.heather@gmail.com

S. R. Feldman

Department of Pathology, Wake Forest School of

Medicine, Winston-Salem, NC, USA

S. R. Feldman

Department of Social Sciences and Health Policy, Wake Forest School of Medicine, Winston-Salem, NC, USA

S. R. Feldman

Department of Dermatology, University of Southern

Denmark, Odense, Denmark therapy for mild-to-severe AD. Therapy is often complex and requires significant patient involvement, which may contribute to poor treatment adherence. Behavioral and social psychology strategies that may help improve adherence include scheduling timely follow-up appointments, using a clearly written eczema action plan (EAP), reducing perceived treatment burden, utilizing anchoring techniques, sharing anecdotes, and rewarding children using positive reinforcement and stickers. There are multiple practical ways by which providers can improve both the management and treatment adherence of patients with AD.

\author{
A. B. Fleischer Jr \\ Department of Dermatology, University of \\ Cincinnati College of Medicine, Cincinnati, $\mathrm{OH}$, \\ USA \\ J. M. Spergel \\ Department of Pediatrics, Perelman School of \\ Medicine at the University of Pennsylvania, \\ Philadelphia, PA, USA \\ J. M. Spergel \\ Division of Allergy and Immunology, Children's \\ Hospital of Philadelphia, Philadelphia, PA, USA \\ E. J. Masicampo · L. K. Kammrath \\ Department of Psychology, Wake Forest University, \\ Winston-Salem, NC, USA
}


Keywords: Adherence; Atopic Behavior; Corticosteroid; Management; Patient; Social Tolerability; Treatment

\section{Key Summary Points}

Atopic dermatitis (AD) is a chronic, inflammatory disease that is characterized by flares of more severe disease states. Diagnosis of AD is largely clinical, and presenting symptoms often includes pruritus, classic morphology, age-specific disease patterns, personal or family history of atopy, and associated comorbidities.

Treatment guidelines include routine use of emollients and trigger-avoidance, topical corticosteroids (TCS), topical nonsteroidal antiinflammatories, humidifiers, phototherapy, and systemic treatments that depend on disease severity.

Current treatment options have the potential to be effective; however, poor treatment adherence amongst patients with $\mathrm{AD}$ hinders disease outcomes.

QD and BID use of TCS yield the same therapeutic outcomes, but QD application may better improve patient adherence to treatment.

Increased frequency of follow-up appointments, clearly written EAPs, and psychology methods such as anchoring and anecdote use can be utilized by providers to improve treatment adherence. The use of adherenceimproving techniques may be as beneficial as developing new drugs to treat AD. dermatitis; Eczema; psychology;

ing

This article is published with digital features, including a summary slide, to facilitate understanding of the article. To view digital features for this article go to https://doi.org/10.6084/ m9.figshare.13688089.

\section{INTRODUCTION}

Atopic dermatitis (AD) is a common inflammatory skin disease that is characterized by eczematous lesions, erythema, and pruritus that affects $15-20 \%$ of children and $1-3 \%$ of adults worldwide $[1,2] . \mathrm{AD}$ is the most common pediatric inflammatory disease in the USA; it is increasing in prevalence and is considered the most burdensome skin disease [3]. Minority populations suffer from greater disease prevalence and severity $[4,5]$. Fortunately, there are new developments in our understanding of $\mathrm{AD}$ and its management.

$\mathrm{AD}$ is often chronic, persistent, and presents in a waxing-and-waning manner, which can be frustrating to patients, their families, and physicians alike [6]. Current AD maintenance strategies and treatment guidelines include routine use of emollients, avoiding triggers, and using regimens that include topical corticosteroids (TCS), nonsteroidal antiinflammatory topicals, phototherapy, and systemic treatments. Although current guidelines outline therapy options that are potentially highly effective, limitations caused by application site discomfort, complicated treatment regimens, and poor adherence hinder AD treatment outcomes in clinical practice $[1,7]$.

Patients often fail to adhere to treatment plans for various reasons including inconvenience of treatment, concerns about side effects, forgetfulness, lack of understanding of their condition, and lack of trust in the provider [8]. The use of topical treatment for AD drops by approximately $70 \%$ within the first few days of beginning a treatment regimen [9]. Various approaches have been developed with the goal of improving the outcome of patients with $\mathrm{AD}$ [10] by addressing the issue of poor treatment adherence and proposing practical approaches 
to improve it $[9,11-14]$. Physicians can use different tools to enhance adherence and outcomes [15]; for example, clearly written treatment plans can help elucidate complex therapy instructions for patients. Also, the use of more frequent follow-ups is an effective motivator towards improved adherence $[16,17]$. Reducing the amount of time between visits or between patient-physician contact (via phone call, for example) decreases the perceived burden of treatment. Adherence depends on patients having a better understanding of $\mathrm{AD}$ and a greater perceived supportive relationship with their provider $[8,15,18]$.

Increased holistic understanding of $\mathrm{AD}$ as a disease-including diagnostic criteria, measurement of severity, and treatment guidelines-as well as the use of clear treatment directions and the incorporation of psychological tools into providers' clinical practice may help improve both $\mathrm{AD}$ management and treatment adherence to topical AD therapies.

\section{METHODS}

An interspecialty, interactive virtual roundtable discussion was held on 19 May 2020 through Wake Forest Department of Dermatology's Annual Spring Meeting Program. Clinical dermatologists, allergists, and behavioral and social psychologists discussed AD treatment, management, and adherence strategies. The information presented during the roundtable was developed from a comprehensive foundation of research and current guidelines that focuses on disease management and treatment adherence of $\mathrm{AD}$. Relevant literature was reviewed, and the content of this article was structured based on the roundtable discussion.

\section{Compliance with Ethics Guidelines}

This article is based on previously conducted studies and does not contain any new studies with human participants or animals performed by any of the authors.

\section{RESULTS}

\section{Overview of AD and Measuring Severity}

\section{Diagnosis and Assessment of $A D$}

The diagnosis of AD is predominantly clinical. Providers observe for specific patterns and morphology, inquire about age of disease onset and known family history, and explore potential associated symptoms. A current guideline for $\mathrm{AD}$ is the revised and adapted Hanifin and Rajka criteria released by the American Academy of Dermatology [2]. The guideline organizes various features of $\mathrm{AD}$ and categorizes them as required features, important features, and associated features necessary for an $\mathrm{AD}$ diagnosis (Fig. 1).

The three required features for assessing and diagnosing a patient with $\mathrm{AD}$ are pruritus, characteristic morphology and age-specific patterns, and chronic or relapsing history. AD morphologies include erythema, edema, papules, excoriation, and lichenification. Typical age-specific patterns seen in patients with $\mathrm{AD}$ include the infantile type, childhood type, and adult type. The infantile pattern of $A D$ often affects the face, scalp, trunk, and extensor surfaces of extremities. The childhood pattern of $\mathrm{AD}$ often presents on the flexural folds of

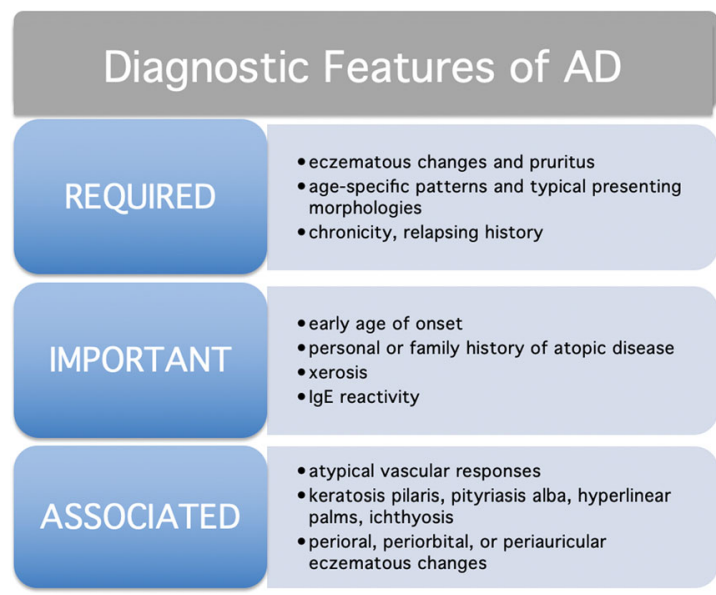

Fig. 1 Diagnosis and assessment of AD. To aid in the diagnosis of $\mathrm{AD}$, the American Academy of Dermatology $(\mathrm{AAD})$ published various features of $\mathrm{AD}$ and categorized them as required features needed for an $\mathrm{AD}$ diagnosis, important features, and associated features [2] 
extensors, around the neck, and ankles. Adulttype $\mathrm{AD}$ most commonly affects the upper arms, back, wrists, hands and fingers, and feet and toes [19]. Due to its chronic and relapsing nature, $\mathrm{AD}$ often persists and presents in a waxing-and-waning manner [6].

The four important features for assessing and diagnosing AD include early age of onset, personal or family history of atopy, xerosis, and IgE reactivity. Onset of AD most commonly presents by 5 years of age, with the highest incidence occurring between 3 and 6 months of age. About $60 \%$ of patients who develop AD do so within the first year of life, while $90 \%$ do so within the first 5 years [2]. Personal or family history of atopy is considered an important feature for the diagnosis of $\mathrm{AD}$ because about $70 \%$ of patients with $\mathrm{AD}$ have a positive family history of atopic diseases [2]. Consequently, the odds of developing $\mathrm{AD}$ are two to three times higher with one atopic parent, rising to a three to five times higher likelihood with two atopic parents [2]. Atopy describes the tendency of a patient to have heightened immune response to common allergens and to develop allergic diseases, which include allergic rhinitis, asthma, and $\mathrm{AD}$. IgE reactivity is considered an important diagnostic feature as $\mathrm{AD}$ is often associated with elevated serum immunoglobulin (IgE) levels. Although elevated IgE is the most commonly associated laboratory finding of $\mathrm{AD}$, such IgE elevation is not present in approximately $20 \%$ of AD-affected patients [2]. Elevated IgE is also nonspecific, because some individuals diagnosed with severe $\mathrm{AD}$ have normal IgE levels, and IgE is elevated in other nonatopic diseases. This laboratory nonspecificity of $\operatorname{IgE}$ is an example of why the diagnosis of $\mathrm{AD}$ remains clinical in practice.

There are many associated features of AD. Atypical vascular responses are commonly observed in patients with $\mathrm{AD}$, including facial pallor, white dermatographism, and a delayed blanch response. Keratosis pilaris, pityriasis alba, palmar plantar hyperlinearity, and ichthyosis are dermatologic conditions often found in conjunction with AD. Regional findings include periorbital, perioral, and periauricular eczematous changes. Other findings associated with $\mathrm{AD}$ and general atopic disease include allergic shiners, Dennie-Morgan folds, and vernal keratoconjunctivitis.

\section{Comorbidities of $A D$}

Common comorbidities among patients with $\mathrm{AD}$ include allergic rhinitis and asthma. Atopic dermatitis, allergic rhinitis, and asthma constitute the classic "allergic triad." Although the conditions do not necessarily occur in conjunction with one another, providers may find it beneficial to ask patients about personal or family history of respiratory diseases like asthma and associated symptoms, such as wheezing and rhonchi, and inquire about environmental and food allergies and associated symptoms including runny nose, watery eyes, itchy throat, urticaria, and anaphylaxis.

Another comorbidity often associated with $\mathrm{AD}$ is allergic contact dermatitis (ACD). Patients with $\mathrm{AD}$ are susceptible to developing ACD at higher rates compared with patients without AD. Allergy or sensitivity to emollients, moisturizers, and topical prescription medications should be considered in clinical practice when treating patients with $\mathrm{AD}$ [20-22].

\section{Methods of Measuring AD Severity}

After diagnosing a patient with $\mathrm{AD}$ using assessment and diagnostic criteria guidelines, various methods can be utilized to measure disease severity. Some of the more commonly used methods include SCORAD, EASI, and vIGA-AD (Fig. 2).

SCORAD (or SCORing Atopic Dermatitis) is a severity measurement method that takes into account affected area, intensity, and subjective symptoms. Severity scores for each category are totaled for a total SCORAD score. The first score category is area, or the sites affected by AD that are typically shaded on a drawing of generic human body in anatomical position. The affected area (AA) can be calculated as a percentage of the total body area (TBA). The percentages of total body area for each region are added up for a total area (A). The second score category is intensity. The provider selects a representative area affected by $\mathrm{AD}$, and the intensity of various categories is measured as none, 0 ; mild, 1 ; moderate, 2 ; or severe, 3 . 


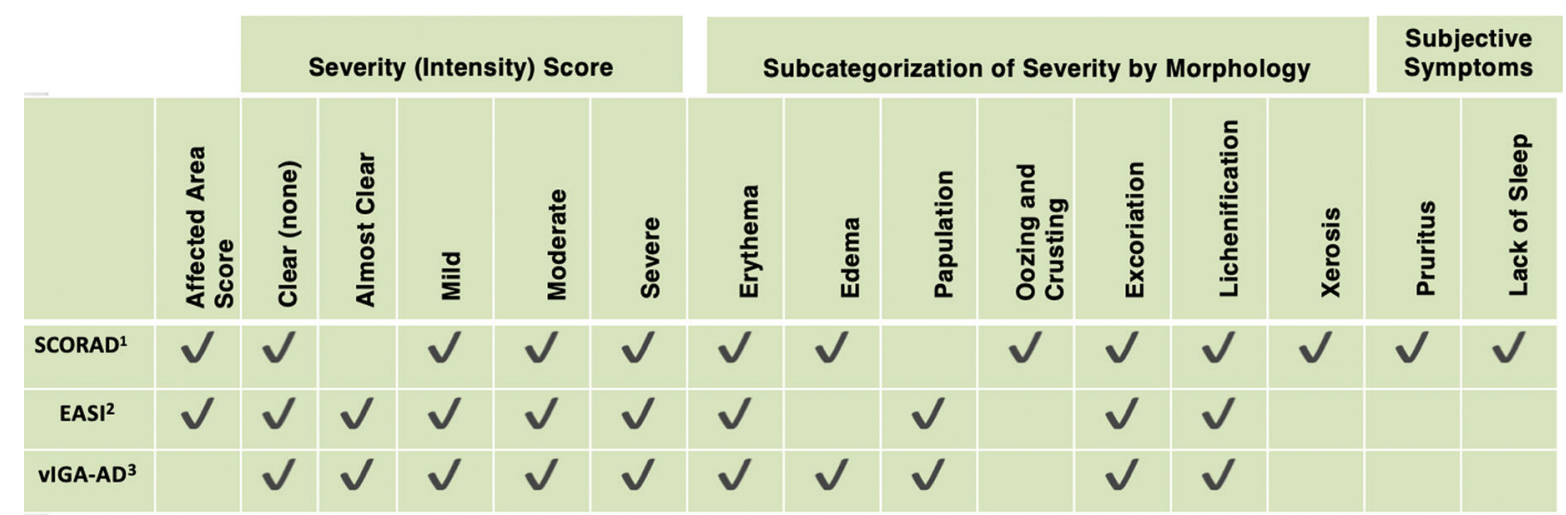

Fig. 2 Comparing AD severity measurement tools. There are various tools that help providers assess $\mathrm{AD}$ disease severity. ${ }^{1}$ SCORing Atopic Dermatitis; ${ }^{2}$ Eczema
Area and Severity Index; ${ }^{3}$ Validated Investigator Global Assessment Scale for Atopic Dermatitis
Intensity scores between zero and three are assigned to six different presenting morphologies: erythema, edema, oozing or crusting, excoriation, lichenification, and xerosis. The intensity score for each morphology is added together for total intensity (B). The final SCORAD category is subjective symptoms, which include pruritus and lack of sleep. The patient measures both pruritus and lack of sleep on a scale of $0-10$, where 0 represents no itch and no sleep loss, and 10 represents worst possible itch and complete sleeplessness. These two subjective symptom scores are added together (C). The final SCORAD total is calculated as $\mathrm{A} / 5+7 \mathrm{~B} / 2+\mathrm{C}$, with a maximum total score of 103.

Another AD severity measurement is the Eczema Area and Severity Index (EASI). Under the EASI system, there are four body regions, and each region has set percentages of total body regions. An area score is recorded for each of the four body regions and comprises the percentage of skin affected by $\mathrm{AD}$ in each region. The area score is scored $0-6$, with 0 being no active $\mathrm{AD}$ in a specific region, and 6 being $90-100 \%$ of the skin affected by AD in that region. Next, severity is scored based on intensity of erythema and inflammation, intensity of papulation, intensity of excoriation, and intensity of lichenification or prurigo nodules. Each severity category is scored on a scale $0-3$, with 0 being none or absent intensity, and 3 being the most severe intensity. The scores for each are added up for a total severity score. To calculate the total EASI score, the total severity score is multiplied by the area score and its "multiplier" for each of the four body regions. Each body region has a unique multiplier value, so the total EASI score is the sum of each multiplication score per body region. The maximum EASI score is 72 , and the minimum is 0 . EASI is the preferred clinimetric approach for severity by Harmonising Outcome Measures for Eczema (HOME).

The vIGA-AD is the Validated Investigator Global Assessment Scale for AD. The vIGA-AD is scored on a scale of 0-4 based on the morphological descriptions including erythema, edema, papules, excoriation, and lichenification. For each presenting morphology: clear, or 0 ; almost clear, or 1 ; mild, or 2; moderate, or 3; and severe, or 4 . The scores 0-4 for each morphology are added together for a total vIGA-AD score.

\section{Stress and AD: A Psychoneuroimmunological Response}

$\mathrm{AD}$ is often accompanied by discomfort that stems from pruritus or pain, sleep disturbance caused by symptoms, and complicated treatment regimens that oftentimes foster stress for both the patient and their family. Stress causes a psychoneuroimmunological response, which exacerbates AD lesions. Psychological stressincluding the stress and decreased quality of life 
caused by uncomfortable symptoms of the condition-upregulates the synthesis of stress hormones such as cortisol. Cortisol has local cutaneous effects by stimulating various proinflammatory responses such as vasodilation and pruritus. Stress hormone mediators also activate mast cells in the skin. The mast cells respond by synthesizing and releasing histamine and proinflammatory molecules including cytokines interleukin (IL)-2, IL-8, IL-31, interferon (IFN) $\gamma$, and tumor necrosis factor (TNF)- $\alpha$. The worsening inflammation and pruritus can intensify a patient's stress, which in turn can propagate the cycle of stress and further exacerbate the inflammation $[22,23]$.

\section{Current AD Treatment Guidelines}

\section{General Guideline Themes for AD Treatment}

$\mathrm{AD}$ treatment regimens suggested by providers may vary depending on the individual patient, taking into account the patient's age, the goal being either maintenance or acute treatment, therapy success in the past, and condition severity. Despite these variations, general themes are shared across different guidelines.

Most treatment guidelines for $\mathrm{AD}$ recommend that patients bathe daily for $5-10 \mathrm{~min}$, followed by moisturizer on both clear and affected skin areas two or three times per day. Topical corticosteroids (TCS) are considered mainstay treatment for $\mathrm{AD}$ and are prescribed following the general "fingertip method" rule. This method emphasizes that $0.5 \mathrm{~g}$ of TCS is enough to cover two palm-sized surfaces on an adult body. The total amount needed per use, however, depends on the total body surface area affected by AD. Application of TCS is generally recommended once or twice per day for acute flare-ups and two or three times per week for maintenance therapy of areas that are prone to flares. Topical calcineurin inhibitors (TCI) are commonly recommended for sensitive areas of the body, including the face and genital region, and are useful if a patient is refractory to TCS or in the case of the steroid-phobic patient. Beyond topical medications, systemic treatment is considered if $\mathrm{AD}$ is resistant to or poorly controlled by topical therapies.
Fig. 3 Categorizing treatment regimens for AD. Managing treatment regimens for $\mathrm{AD}$ can be effectively organized based on severity (clear or nonlesional, mild, moderate, and severe), and further categorized by maintenance (daily regimen for clear skin) treatments and acute (flare-up) treatments in patient-friendly language [20]

\section{AD Treatment Guidelines: Acute, Maintenance, Severity}

Organizing treatment guidelines in a clear, understandable manner for patients to follow may be beneficial to both patients and providers alike, as $\mathrm{AD}$ therapy is often complex and involved.

Guidelines are categorized by severity under nonlesional versus mild versus moderate versus severe $\mathrm{AD}$, and further subcategorized as acute flare-up treatment versus maintenance treatment. Information from the $\mathrm{AD}$ yardstick for practical treatment recommendations [20] was reconfigured and presented in a patient-friendly language that may be valuable in clinical practice (Fig. 3).

Maintenance therapy for nonlesional AD includes a daily skin care regimen and trigger avoidance. A maintenance skin care routine consists of liberal and frequent moisturizer use. Guidelines recommend that patients take daily, warm 5-10 min baths or showers and use nonsoap cleansers, directly followed by moisturizer application. Moisturizer can be applied to both problem areas and nonlesional areas of the skin. Common allergens and irritants should be avoided; these include - but are not limited tosoaps, fragrance, wool, and temperature extremes. Comorbidities of AD should also be considered, such as asthma, allergic rhinitis, and ACD.

Maintenance therapy for mild AD includes daily, liberal moisturizing (especially after bathing), and trigger avoidance. Antiseptic measures and humidifier use should also be implemented. Recommended antiseptic measures include a dilute bleach bath, which consists of one capful of bleach into one bathtub filled with water, one-to-two times per week. Antiseptic measures are especially useful if the patient suffers from recurrent skin infections. Antibiotics can also be considered to treat skin 


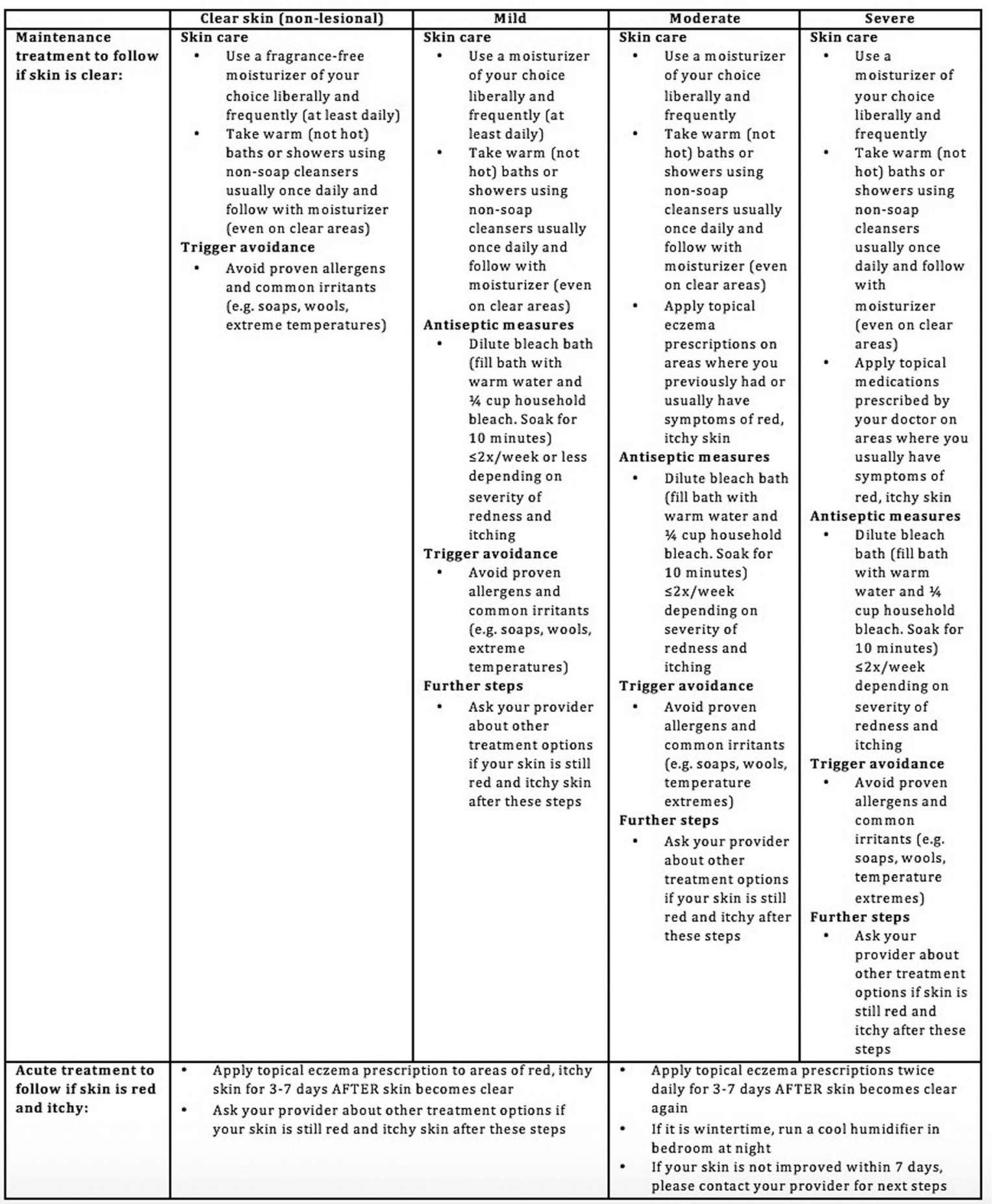


infection, if necessary. Humidifier use is also recommended, especially during the dry, cold winter months.

Maintenance therapy for moderate AD includes daily skin moisturizing, trigger avoidance, antiseptic measures, and humidifier use, similar to maintenance therapy for mild AD. In addition to this basic management, topical antiinflammatory medication may be introduced here. Maintenance low-potency TCS can be used once or twice per day. Medium-potency TCS for moderate AD maintenance therapy is recommended once or twice weekly, excluding the face. If a patient does not respond well to TCS, providers can suggest using maintenance TCI once or twice a day, or two to three times per week as needed. Crisaborole $2 \%$ can also be used twice daily as maintenance for moderate AD but is not considered first-line therapy. Patients with moderate AD may consider wet wrap therapy as well.

Maintenance therapy for severe AD includes daily skin moisturizing, trigger avoidance, antiseptic measures, humidifier use, and antiinflammatory topicals. In addition to this basic management, providers may consider referring the patient to an $\mathrm{AD}$ specialist. Additional maintenance treatment options for patients with severe $\mathrm{AD}$ include phototherapy, biologics such as dupilumab, or systemic immunosuppressants such as cyclosporine A, methotrexate, mycophenolate mofetil, azathioprine, and oral corticosteroids.

In addition to maintenance therapies across all disease severities, $\mathrm{AD}$ treatment guidelines outline ways that can help patients gain control during acute flare-ups. Acute treatment for nonlesional and mild AD includes applying TCS only to areas of inflamed skin. Low- to mediumpotency TCS applied twice daily for 3-7 days beyond clearance is recommended for acute therapy. Providers can also consider prescribing TCI or crisaborole for sensitive areas such as the face or genitals, or if patients are refractory to treatment using TCS.

Acute treatment for moderate-to-severe $\mathrm{AD}$ includes applying a medium- to high-potency TCS twice daily for 3-7 days beyond clearance. TCI and crisaborole can be considered for sensitive areas or patients who are not responsive to TCS therapy. If the acute inflammation does not resolve in 7 days, providers should consider other factors including treatment nonadherence, infection, misdiagnosis, or development of contact allergy to medication. Providers may also consider referring to an AD specialist. Firstgeneration oral antihistamines are recommended for pruritus control and associated sleep disturbances. Other treatment options that may help patients gain control of severe, acute $\mathrm{AD}$ flare-ups include wet wrap therapy and short-term hospitalization, especially in the case of infection. Maintenance and acute therapy options across $\mathrm{AD}$ severities can be organized and presented to patients in a clear manner that is easy to follow (Fig. 3).

\section{Stepping-Up AD Therapy}

As AD usually presents in a waxing-and-waning pattern, it is essential for providers to understand when it is deemed appropriate to change treatment regimens, assess for nonadherence, and how to step up therapy if a patient's condition worsens (Fig. 4).

For patients with mild AD that has exacerbated to a moderate degree of severity, patients usually remain symptomatic despite low- to medium-potency TCS use and maintenance skin care regimens. Guideline treatment options for patients stepping up from mild to moderate AD suggest increasing the TCS dose or potency, adding a TCI, or adding crisaborole $2 \%$ ointment. The treatment change suggested by the provider is trialed for about 3 months, with a follow-up appointment scheduled within the first 1 or 2 months to assess for progress and troubleshoot any issues such as contact allergy, application difficulty, or nonresponsiveness [20].

Patients with moderate AD who worsen to severe disease may have issues with mental health, lower quality of life, and impaired daily activities. Guidelines suggest alternative treatment options including phototherapy, dupilumab, or systemic therapies such as cyclosporine, methotrexate, mycophenolate mofetil, azathioprine, or oral corticosteroids. Providers may also suggest that patients see an $\mathrm{AD}$ specialist [20]. 

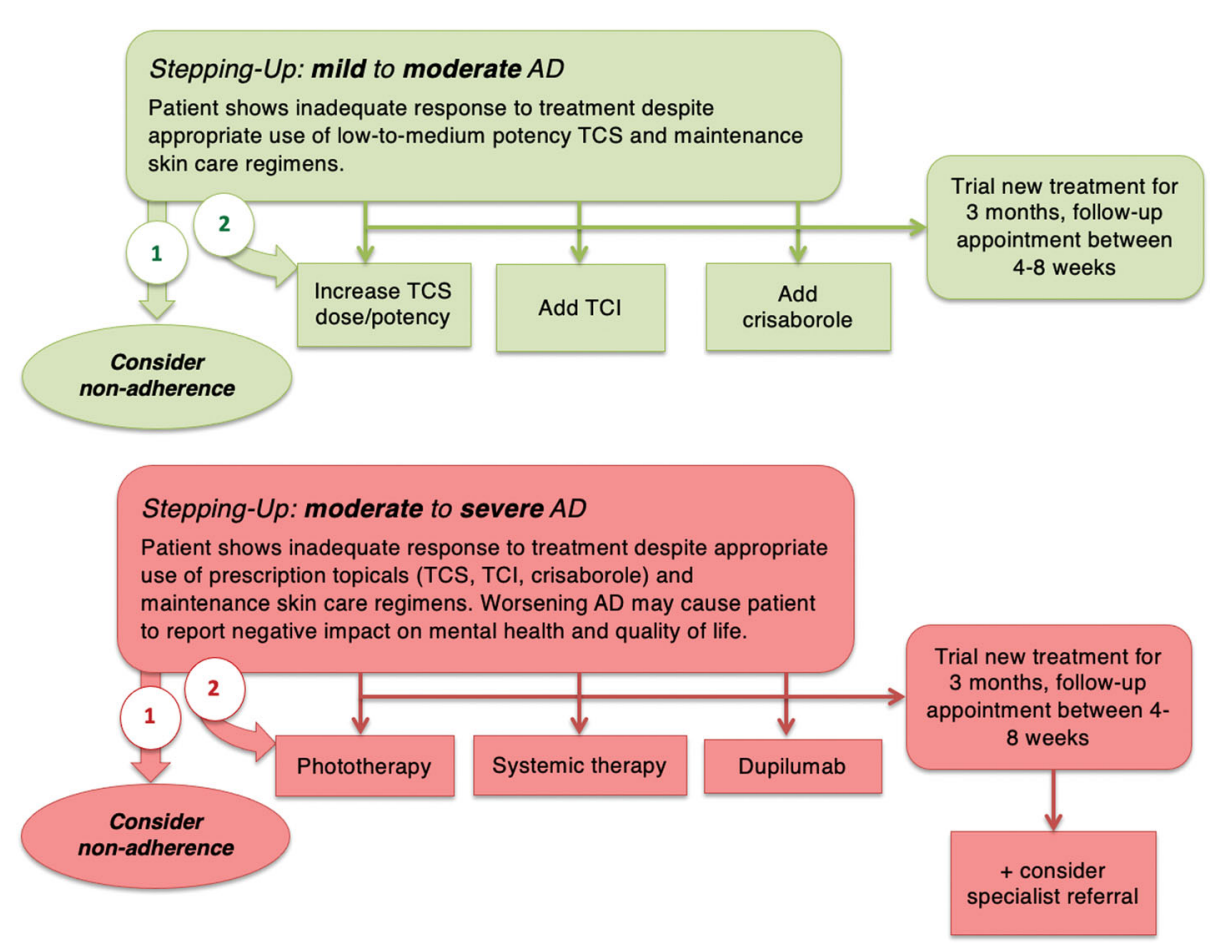

Fig. 4 Step-up treatment for worsening AD. Organization of guidelines depicts when it may be appropriate to change treatment regimens or how to step up therapy if

Before initiating new treatment regimens, however, it may be beneficial to consider the patient's adherence to treatment-or lack thereof. Treatment adherence among patients with $\mathrm{AD}$ is poor, and a patient's adherence is often an unknown factor. Most patients do not use medications as recommended [1, 7]. Without primarily assessing for treatment adherence, providers may be unnecessarily changing or complicating treatment regimens while the reason for unresponsiveness or disease exacerbation-poor adherence-remains unresolved. Although it is possible to initiate new therapies when the patient is thought to be fully adherent yet fails to see improvement in their condition, it is more likely that patients are nonadherent to recommended mainstay treatment. Without inquiring about and working to improve adherence, patients may be at risk for unnecessary side effects that could be avoided if mainstay topical treatments were used properly. Specific techniques that may help improve
AD worsens [20]. Treatment adherence should be adequately assessed before initiating new therapy or introducing systemic treatment

patient adherence to $\mathrm{AD}$ treatment are discussed in a later section.

\section{Systemic Therapies, New Treatments, and Biologics}

Although skin care maintenance regimens and topical antiinflammatory medications are mainstay AD treatments, high-severity disease may require systemic therapies for relief. Cyclosporine, methotrexate (MTX), mycophenolate mofetil, and azathioprine are systemic treatment options that providers may suggest. These systemic therapies, however, pose certain risks and side effects. Most systemic AD treatments are immunosuppressive and increase risks of infection, and may cause renal and hepatic toxicity. Guidelines suggest that providers increase the potency or frequency of topical medications, confirm and improve patient adherence to maintenance skin care regimens, and utilize humidifiers, bleach baths, or wet wrap therapies before implementing additional systemic therapeutics. 
Novel treatment options for AD available for clinical use include crisaborole and dupilumab. Crisaborole is a topical, nonsteroidal phosphodiesterase- 4 inhibitor that is approved to treat mild-to-moderate $\mathrm{AD}$ in patients 2 years of age and older [24]. Dupilumab is an IL-4 receptor monoclonal antibody that binds to the alpha chain of the IL-4 receptor and inhibits downstream inflammation often seen in atopic diseases such as AD. Dupilumab improves lesion healing, pruritus, and sleep quality compared with placebo [24], and is approved for patients older than 12 years of age with moderate-tosevere AD who previously failed topical therapies [21].

\section{Techniques to Improve Treatment Adherence}

\section{The Downward Trend of AD Treatment} Adherence

Patient adherence to topical treatment is poor, and reasons for nonadherence include lack of understanding of the condition, inconvenience of treatment, forgetfulness, concerns about side effects, and lack of trust in the provider [8]. If patients are not responding to treatment, poor adherence should be considered and appropriate adherence-promoting measures can be instituted.

Adherence to topical treatment for AD drops by approximately $70 \%$ within the first week of starting a treatment regimen [9]. The rates of adherence continue to drop with time (Fig. 5). The timing of follow-up appointments can be used to improve adherence [16, 17]. While treatment adherence generally decreases with time, anticipation of a scheduled follow-up increases adherence rates before the scheduled appointment (Fig. 5). After a follow-up appointment, adherence decreases again. Anticipation of visits or other forms of patient-physician contact may increase a patient's sense of accountability and, consequently, treatment adherence.

\section{Adherence Intervention Pyramid}

The adherence intervention pyramid [10] organizes techniques that can be utilized by providers to help improve treatment adherence

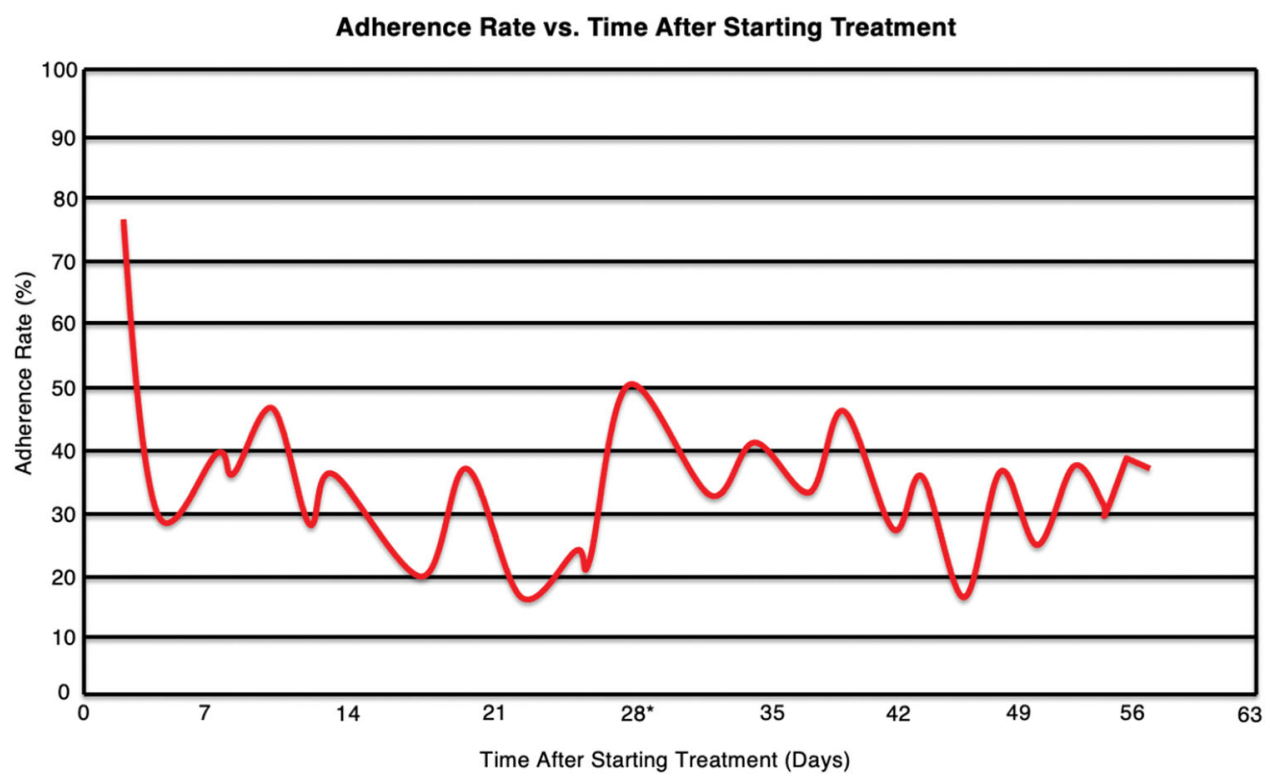

Fig. 5 Average rate of adherence versus time after starting topical treatment. Adherence to topical treatment is poor, and average rates of adherence decline as time passes after the initial appointment and start of treatment. Adherence tends to increase just before a scheduled follow-up appointment $\left(^{*}\right)$, but then declines again. More frequent follow-ups may be beneficial in helping to improve adherence to therapy in $\mathrm{AD}$ [9] 


\section{Adherence Intervention Pyramid}

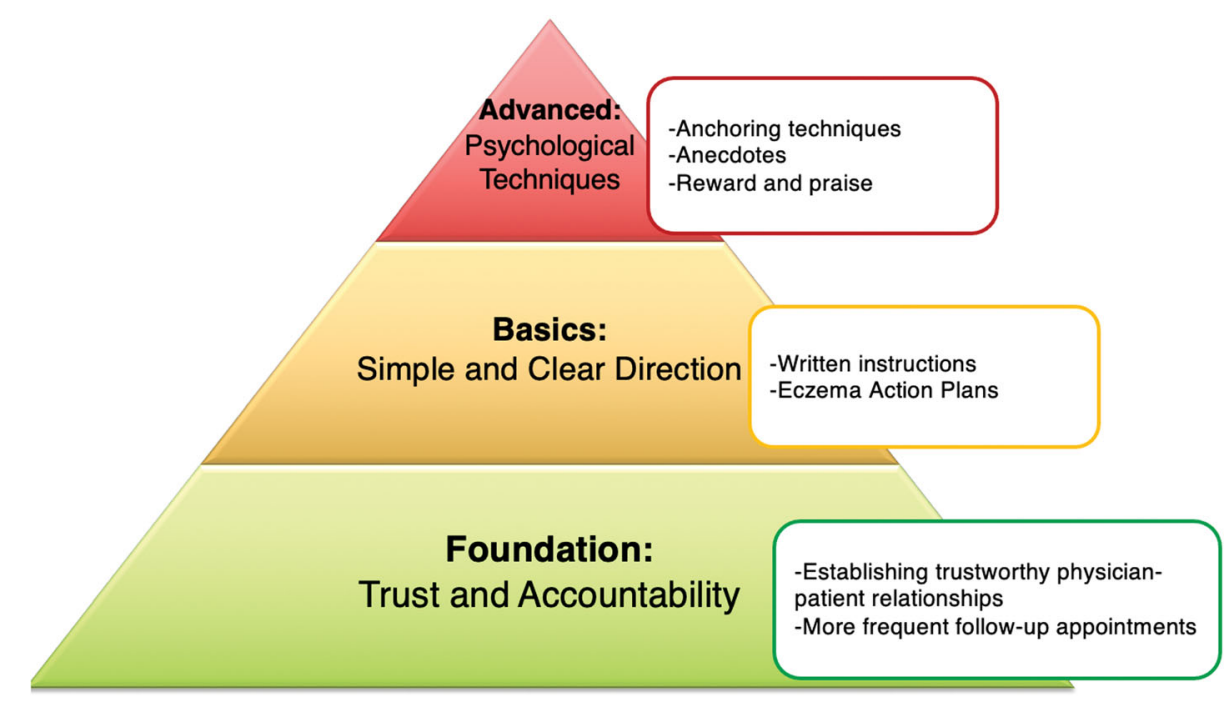

Fig. 6 Adherence intervention pyramid. The groundwork for helping to improve patient treatment adherence starts with establishing a trusting physician-patient relationship and scheduling more frequent follow-up appointments. These follow-ups and more frequent interactions with the patient will not only help to deepen the

among patients with AD (Fig. 6). The foundation of the pyramid encompasses trust and accountability. The next level of the pyramid focuses on common, recommended ways to improve adherence such as giving patients simple treatment plans with clear, written direction, motivating patients, and considering costs of treatment. The top of the pyramid consists of advanced psychological techniques such as anchoring, the use of anecdotes, and utilizing rewards and praise.

\section{Trust and Accountability}

Establishing a trusting physician-patient relationship is essential, as treatment adherence improves with an increase in perceived support from the prescribing provider $[8,15,18]$. The importance of establishing accountability is easily understood via a piano lesson analogy: a piano teacher gives music sheets to a student on the first day and instructs the student to practice every day for a recital that will take place in a few months. In one scenario, the sheet music physician-patient relationship but also improve patient accountability. Another step to improving treatment adherence is to provide clear, written instruction for patients in the form of an EAP. Psychological techniques can also be employed, including the use of anchoring, providing anecdotes, and using rewards and praise [10]

is given on day one, and the teacher has weekly piano lessons with the student in preparation for the upcoming recital. The recital is a success because the students practiced weekly at the lessons. In an opposing scenario, the sheet music is given on the first day, but there are no weekly piano lessons with the piano teacher. The student arrives at the recital unprepared, and the recital is a disaster. The idea that the latter approach would be successful is laughable; only in medicine do we give people instructions to do something every day and not check in on them for months thereafter [24].

\section{Approaches for Improving Adherence}

The middle of the pyramid focuses on standard, commonly recommended approaches to improve adherence including simple treatment plans, written instruction, affordable medications, motivational interviewing, and patient education. Patient education and clear, written instruction are essential for improving treatment adherence. An eczema action plan (EAP) is 
a written instruction of a patient's treatment regimen put together by the provider, which helps to elucidate complex instructions and organizes the use of the products and techniques that typically compose complicated, multistep AD therapy [25].

A step beyond a generic EAP is the "pseudotailored" EAP. Since AD treatment outcomes are often limited by poor adherence to topical treatments and maintenance recommendations, "pseudotailoring" techniques can help increase a patient's attention to their treatment plan, with the goal of improving overall adherence. Through the utilization of pseudotailored EAPs, expectations of customization can be raised without actually providing individualized content [26]. Although the treatment plan is not truly personalized, the patient often perceives that it is. A survey study analyzed the effect of pseudotailored $\mathrm{AD}$ treatment plans on overall patient satisfaction, confidence in their treatment plan, and trust in their provider. One group received a generic EAP (Fig. 7), while the other group received a pseudotailored EAP with circled treatment selections among decoy options (Fig. 8). Pseudotailoring improved overall patient satisfaction in the treatment plan, perception of personalization, and willingness to adhere to the recommended treatment regimen [26].

\section{More Advanced Psychological Techniques}

The top of the pyramid focuses on implementing psychological techniques such as anchoring, anecdotes, and rewards and praise.

Anchoring is a psychological technique that utilizes cognitive bias in decision-making. It describes the human tendency to rely on-or "anchor"-on a specific piece of information as a reference or starting point. This technique is better comprehended through the use of examples; regarding improving $\mathrm{AD}$ treatment adherence, a provider may begin by explaining to a patient that a topical medication needs to be applied four times daily. The provider then "compromises" with the patient and instructs them to only apply the topical twice daily. This perceived smaller burden due to the use of anchoring increases adherence and willingness to properly follow treatment instructions.

Another example of anchoring is asking patients how willing they are to inject a subcutaneous medication indicated once monthly. A provider can start by first asking how willing the patient is to inject once a day, and once the anchor is in place, the provider asks how willing

Generic Treatment Plan

\begin{tabular}{|l|l|}
\hline \multicolumn{2}{|c|}{ Your Treatment Plan } \\
\hline Moisturizer: & Emollient \\
Topical Corticosteroid: & Triamcinolone \\
Topical Corticosteroid Frequency: & Two (2) times per day \\
Bath: & Diluted bleach \\
Diet: & Anti-inflammatory \\
Other: & Humidifier \\
\hline Details: \\
\hline Diluted bleach bath: Add $1 / 4$ cup of household bleach to a half-full bathtub of water. Soak \\
for 5-10 minutes. Rinse completely with warm (not hot) water. \\
Anti-inflammatory diet: Avoid foods high in saturated fats, refined grains, and processed \\
meats. Consume foods high in omega-3 (fish i.e. salmon), probiotics (i.e. yogurt with active \\
cultures), and colorful fruits and vegetables. \\
\hline
\end{tabular}

Fig. 7 Generic treatment plan. An example of a generic EAP, which provides written instruction for a patient's treatment regimen to clarify complex directions and organize products and techniques used in typical, multistep AD therapy [26] 
Pseudotailored Treatment Plan

\begin{tabular}{|c|c|c|}
\hline \multicolumn{3}{|c|}{ Your Treatment Plan } \\
\hline $\begin{array}{l}\text { Moisturizer: } \\
\text { Topical Corticosteroid: } \\
\text { Topical Corticosteroid Frequency: } \\
\text { Bath: } \\
\text { Diet: } \\
\text { Other: }\end{array}$ & \begin{tabular}{|l} 
Emollient \\
Hydrocortisone \\
1x/day \\
Oatmeal \\
Dairy-free \\
Humidifier \\
\end{tabular} & $\begin{array}{ll}\text { Humectant } & \text { Occlusive } \\
\text { Betamethasone } & \text { Triamcinolone } \\
\text { 2x/day } & \text { 4x/day } \\
\text { Diluted bleach } & \text { Regular } \\
\text { Gluten-free } & \text { Anti-inflammatory } \\
\text { Anti-histamines } & \text { Wet-wrap }\end{array}$ \\
\hline $\begin{array}{l}\text { Details: } \\
\text { Oatmeal bath: Add } 2-3 \text { cups colloidal } \\
\text { Rinse completely with warm (not hot) } \\
\text { Diluted bleach bath: Add } 1 / 4 \text { cup of } h \\
5-10 \text { minutes. Rinse completely with v } \\
\text { Dairy-free diet: Avoid foods containin } \\
\text { Gluten-free diet: Avoid foods contain } \\
\text { Anti-inflammatory diet: Avoid foods } \\
\text { meats. Consume foods high in omega }\end{array}$ & $\begin{array}{l}\text { oatmeal to a bat } \\
\text { water. } \\
\text { ousehold bleach } \\
\text { Narm (not hot) we } \\
\text { ig lactose, whey, } \\
\text { ing wheat, barley } \\
\text { high in saturated } \\
\text { a-3 (fish i.e. salm } \\
\text { tables. }\end{array}$ & $\begin{array}{l}\text { thtub full of water. Soak for } 10 \text { minutes. } \\
\text { to a half-full bathtub of water. Soak for } \\
\text { ater. } \\
\text { casein, cheese powder, lactalbumin. } \\
\text { y, rye, oats, bulgur. } \\
\text { fats, refined grains, and processed } \\
\text { on), probiotics (i.e. yogurt with active }\end{array}$ \\
\hline
\end{tabular}

Fig. 8 Pseudotailored treatment plan. An example of a pseudotailored EAP, which provides the experience of customization without actually providing individualized

the patient would be to inject once per month instead. Willingness to adhere to treatment was approximately four times higher in patients who were anchored than those who were not (Fig. 9) [27].

Another example of anchoring in combination with the ability to simultaneously lower the perceived burden of treatment is by explaining to patients how moisturizers alone will take up to 6 weeks to see improvement. It is then explained that, with the addition of TCS, the patient may see improvement in only 1 or 2 weeks. This technique may be more effective than simply describing how TCS takes 1 or 2 weeks to see improvement alone. One or 2 weeks may be perceived as a long time for a patient without context. An anchor provides context that makes those 1 or 2 weeks seem much shorter, thus decreasing the burden of treatment and increasing adherence.

Another psychological technique that may help improve treatment adherence while simultaneously improving the physician-patient relationship is the use of anecdotes in content. The treatment plan is perceived as personalized by the patient. Treatment options are circled among decoy options [26]

clinical practice. Human beings are more influenced by things they can imagine and picture as reality. Numbers and data alone are less influential and less effective than personal stories and examples [28]. Stories make patients feel more comfortable than data. Providers who share personal experiences or previous patient success stories with their patients-i.e., "I had a patient with a very similar case to yours who did very well on this treatment" - are more likely to instill trust and confidence into their patients, and improve treatment adherence to the prescribed regimen.

Another psychological technique that can be used to improve treatment adherence is the implementation of rewards and praise, especially amongst pediatric patients. Positive reinforcement and the use of stickers or sticker charts to track a patient's treatment progress is an effective way to instill enthusiasm in children regarding treatment adherence and lower the burden for parents or family members [10].

The adherence intervention pyramid incorporates a range of valuable methods and tools 


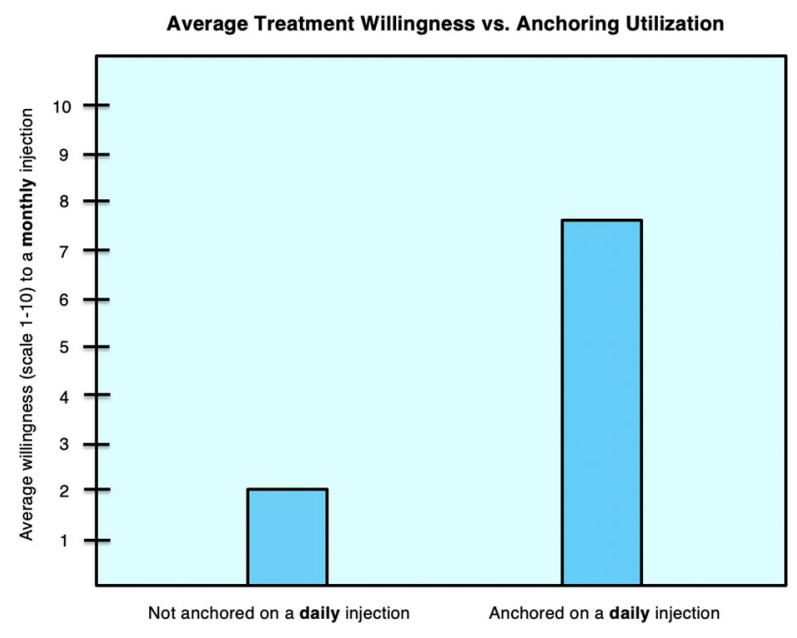

Fig. 9 Effect of anchoring on patient treatment willingness. Patients were split into two groups and asked about their willingness to inject a subcutaneous medication indicated once monthly. The first group was not anchored, and was only asked about their willingness to inject once monthly. The second group was anchored by first being asked how willing they were to inject daily. After being asked about once daily injections, the anchor group was then asked how willing they were to inject once monthly. The willingness was scored on a scale of $1-10$, with 1 being not at all willing, and 10 being the most willing. Average willingness to adhere to treatment was almost four times higher in patients who were anchored than those who were not [27]

that can be utilized to help improve treatment adherence and benefit patients with AD.

\section{DISCUSSION}

\section{Promising New Therapies}

Although the mainstay treatment for $\mathrm{AD}$ is maintenance therapy, there are novel drugs in development that may be promising new options for patients with AD. Treatments currently undergoing clinical trials, but that are not yet on the market for clinical use, include anti-leukin-31 receptor antagonists, IL-13 monoclonal antibody drugs, histamine-4 receptor (H4R) antagonists, and systemic and topical Janus kinase (JAK) inhibitors.

Anti-interleukin-31 receptor antagonists include nemolizumab (CIM331), a humanized monoclonal antibody against IL-31 receptor A. The antibody binds to IL-31 receptor A to inhibit IL-31 signaling. IL-31 receptors are commonly found on neurons, and nemolizumab helps alleviate pruritus [29].

Tralokinumab and lebrikizumab are novel, monoclonal antibody drugs that bind IL-13 to prevent the formation of the IL-13R $\alpha 1 / \mathrm{IL}-4 \mathrm{R} \alpha$ complex and downstream signaling, thus blocking the inflammatory effects of IL-13 seen in atopic conditions such as AD.

Histamine-4 receptor antagonists include pyrido[2,3-e]tetrazolo[1,5-a]pyrazine analogue 48 , a selective H4R antagonist. Orally administered, it is antipruritic and antiinflammatory, which is promising for clinical treatment among patients with $\mathrm{AD}[30,31]$.

JAK inhibitors include baricitinib, an oral JAK inhibitor that is highly selective for JAK1 and JAK2. Treatment with baricitinib improved the signs and symptoms of moderate-to-severe AD compared with placebo [32]. Other novel systemic, JAK-targeting agents include abrocitinib and upadacitinib, which are both oral JAK1-selective inhibitors. Novel topical JAKtargeting therapies include ruxolitinib and delgocitinib. Ruxolitinib is a selective inhibitor of JAK1 and JAK2 that suppresses the signaling of cytokines involved in AD. Delgocitinib is a panJAK inhibitor that blocks JAK1, JAK2, JAK3, and tyrosine kinase 2 signaling pathways, thus inhibiting the inflammation seen in AD.

\section{Improving Treatment Adherence: Understanding the Value}

AD can detrimentally impact quality of life for patients and their families. Pruritus and excoriation often lead to sleep disturbances; emotional detriments include irritability, behavioral issues, embarrassment, and social isolation [7]. Physical, emotional, and social detriments increase stress levels, which, in turn, further exacerbate AD.

$\mathrm{AD}$ treatments can also be cost-prohibitive for patients, especially newer treatment options that have recently appeared on the market. General disease costs include prescriptions, over-the-counter products, physician 
appointments, and hospitalizations. If treatment adherence to the more cost-effective treatment options-such as mainstay moisturizers and TCS-is optimized, it may provide economic relief to patients and their families. Even novel, effective therapies such as dupilumab are only as effective as the patient is adherent.

Improving adherence is essential for optimizing disease outcomes, which in turn improves quality of life and reduces economic burden. Developing and utilizing techniques to improve adherence may be as beneficial as-and possibly more achievable, cost-effective, and practical than-developing new drugs to treat AD. Research that aims to elucidate the patterns of nonadherence among patients with $\mathrm{AD}$ and the methods that help improve it is well documented [33]. What is most needed is better education amongst providers who treat $\mathrm{AD}$ on treatment adherence strategies and how they can effectively implement them into clinical practice.

\section{ACKNOWLEDGEMENTS}

Funding. Sponsorship for this study was funded by Pfizer Inc. Pfizer Inc. New York, NY USA. The Pfizer funds awarded to the institution included the funds for publication fees.

Authorship. All named authors meet the International Committee of Medical Journal Editors (ICMJE) criteria for authorship for this article, take responsibility for the integrity of the work as a whole, and have given their approval for this version to be published.

Disclosures. Dr. Steven Feldman has received research, speaking and/or consulting support from a variety of companies including Galderma, GSK/Stiefel, Almirall, Leo Pharma, Boehringer Ingelheim, Mylan, Celgene, Pfizer, Valeant, Abbvie, Samsung, Janssen, Lilly, Menlo, Merck, Novartis, Regeneron, Sanofi, Novan, Qurient, National Biological Corporation, Caremark, Advance Medical, Sun Pharma, Suncare Research, Informa, UpToDate and
National Psoriasis Foundation. He is founder and majority owner of http://www.DrScore.com and founder and part owner of Causa Research, a company dedicated to enhancing patients' adherence to treatment. Dr. Alan Fleischer is a consultant for Boerhringer-Ingelheim, Incyte, Qurient, SCM Lifescience, Syneos and Trevi. He is an investigator for Galderma and Trevi. The authors otherwise declare no conflicts of interests. Heather L. Tier, Esther A. Balogh, Arjun M. Bashyam, Jonathan M. Spergel, EJ Masicampo, Lara K. Kammrath and Lindsay C. Strowd have nothing to disclose.

Compliance with Ethics Guidelines. This article is based on previously conducted studies and does not contain any new studies with human participants or animals performed by any of the authors.

Data Availability. Data sharing is not applicable to this article as no datasets were generated or analyzed during the current study.

Open Access. This article is licensed under a Creative Commons Attribution-NonCommercial 4.0 International License, which permits any non-commercial use, sharing, adaptation, distribution and reproduction in any medium or format, as long as you give appropriate credit to the original author(s) and the source, provide a link to the Creative Commons licence, and indicate if changes were made. The images or other third party material in this article are included in the article's Creative Commons licence, unless indicated otherwise in a credit line to the material. If material is not included in the article's Creative Commons licence and your intended use is not permitted by statutory regulation or exceeds the permitted use, you will need to obtain permission directly from the copyright holder. To view a copy of this licence, visit http://creativecommons.org/licenses/by$\mathrm{nc} / 4.0 /$. 


\section{REFERENCES}

1. Draelos ZD, et al. Tolerability of topical treatments for atopic dermatitis. Dermatol Therapy. 2019;9(1): 71-102.

2. Eichenfield LF, et al. Guidelines of care for the management of atopic dermatitis: Section 1. Diagnosis and assessment of atopic dermatitis. J Am Acad Dermatol. 2014;70(2):338-51.

3. Hay RJ, et al. The global burden of skin disease in 2010: an analysis of the prevalence and impact of skin conditions. J Investig Dermatol. 2014;134(6): 1527-34.

4. Silverberg JI. Public health burden and epidemiology of atopic dermatitis. Dermatol Clin. 2017;35(3): 283-9.

5. Hsu DY, Shinkai K, Silverberg JI. Epidemiology of eczema herpeticum in hospitalized US children: analysis of a nationwide cohort. J Investig Dermatol. $2018 ; 138(2): 265-72$.

6. Margolis JS, et al. Persistence of mild to moderate atopic dermatitis. JAMA Dermatol. 2014;150(6): 593-600.

7. Feldman SR, et al. The challenge of managing atopic dermatitis in the United States. Am Health Drug Benefits. 2019;12(2):83.

8. Patel NU, D'Ambra V, Feldman SR. Increasing adherence with topical agents for atopic dermatitis. Am J Clin Dermatol. 2017;18(3):323-32.

9. Krejci-Manwaring J, et al. Stealth monitoring of adherence to topical medication: adherence is very poor in children with atopic dermatitis. J Am Acad Dermatol. 2007;56(2):211-6.

10. Lewis D, Feldman S. Practical ways to improve patient adherence. Middletown: CreateSpace Independent Publishing Platform; 2017.

11. Conde JF, et al. Adherence to clocortolone pivalate cream $0.1 \%$ in a pediatric population with atopic dermatitis. Cutis. 2008;81(5):435-41.

12. Brown KL, Krejci-Manwaring J, Tusa MG, Camacho F, Fleischer AB Jr, Balkrishnan R, Feldman SR. Poor compliance with topical corticosteroids for atopic dermatitis despite severe disease. Dermatol Online J. 2008;14(9):13.

13. Yentzer BA, et al. Good adherence and early efficacy using desonide hydrogel for atopic dermatitis: results from a program addressing patient compliance. J Drugs Dermatol JDD. 2010;9(4):324-9.
14. Ou H-T, Feldman SR, Balkrishnan R. Understanding and improving treatment adherence in pediatric patients. Semin Cutan Med Surg. 2010;29(2): 137-40.

15. Feldman SR, et al. Treatment adherence intervention studies in dermatology and guidance on how to support adherence. Am J Clin Dermatol. 2017;18(2):253-71.

16. Sagransky MJ, et al. A randomized controlled pilot study of the effects of an extra office visit on adherence and outcomes in atopic dermatitis. Arch Dermatol. 2010;146(12):1428-31.

17. Feldman SR, et al. Adherence to topical therapy increases around the time of office visits. J Am Acad Dermatol. 2007;57(1):81-3.

18. Kim HB, et al. Survey on the management of atopic dermatitis. Korean J Asthma Allergy Clin Immunol. 2012;32(1):16-20.

19. Weston W, Lane A, Morelli J. Color textbook of pediatric dermatology. 4th ed. Philadelphia: Mosby Elsevier; 2007.

20. Boguniewicz M, et al. Atopic dermatitis yardstick: practical recommendations for an evolving therapeutic landscape. Ann Allergy Asthma Immunol. 2018;120(1):10-22.e2.

21. Sastre J, Davila I. Dupilumab: a new paradigm for the treatment of allergic diseases. J Investig Allergol Clin Immunol. 2018;28(3):139-50.

22. Buddenkotte J, Steinhoff M. Pathophysiology and therapy of pruritus in allergic and atopic diseases. Allergy. 2010;65(7):805-21.

23. Suárez AL, et al. Psychoneuroimmunology of psychological stress and atopic dermatitis: pathophysiologic and therapeutic updates. Acta Derm Venereol. 2012;92(1):7-15.

24. Feldman S. The parable of the piano teacher. The Dermatologist. 2007;15(8).

25. Rork JF, et al. Parental response to written eczema action plans in children with eczema. Arch Dermatol. 2012;148(3):391-2.

26. Bashyam AM, et al. Placebo tailoring improves patient satisfaction of treatment plans in atopic dermatitis. J Am Acad Dermatol. 2020;83:944-6.

27. Oussedik E, et al. An anchoring-based intervention to increase patient willingness to use injectable medication in psoriasis. JAMA Dermatol. 2017;153(9):932-4. 
28. Feldman SR, et al. Treatment adherence in dermatology. Cham: Springer; 2019.

29. Ruzicka T, et al. Anti-interleukin-31 receptor A Antibody for atopic dermatitis. $\mathrm{N}$ Engl J Med. 2017;376(9):826-35.

30. Schaper-Gerhardt K, et al. The role of the histamine $\mathrm{H}(4)$ receptor in atopic dermatitis and psoriasis. $\mathrm{Br} \mathrm{J}$ Pharmacol. 2020;177(3):490-502.

31. Ko K, et al. Discovery of a novel highly selective histamine $\mathrm{H} 4$ receptor antagonist for the treatment of atopic dermatitis. J Med Chem. 2018;61(7): 2949-61.

32. Napolitano $\mathbf{M}$, et al. Profile of baricitinib and its potential in the treatment of moderate to severe atopic dermatitis: a short review on the emerging clinical evidence. J Asthma Allergy. 2020;13:89-94.

33. Bass AM, Anderson KL, Feldman SR. Interventions to increase treatment adherence in pediatric atopic dermatitis: a systematic review. J Clin Med. 2015;4(2):231-42. 